\title{
Différences de sensibilité à Matsucoccus feytaudi DUC (Homoptera : Margarodidae) selon les provenances de pin maritime (Pinus pinaster AIT)
}

\author{
D. SCHVESTER, F. UGHETTO \\ INRA, Station de Zoologie forestière \\ avenue A.-Vivaldi, F 84000 Avignon
}

\begin{abstract}
Résumé
Observations sur le comportement à l'infestation par Matsucoccus feytaudi DUC. de diverses provenances de pin maritime (Pinus pinaster AIT.), dans une plantation comparative mise en place en 1965 dans le Massif des Maures.

Les observations ont consisté en :

- notations symptomatologiques arbre par arbre, poursuivies de 1979 à 1984 ;

- une recherche d'explication des différences observées au moyen, d'une part d'une évaluation des niveaux de population d'insectes (par piégeages et par comptages), et d'autre part, sur certaines provenances, par un examen des caractères des tissus corticaux.

Les notations sur les symptômes (tabl. 1 et fig. 1) mettent en évidence de grandes différences dans la sensibilité des provenances testées, à $M$. feytaudi. différences.

Différentes hypothèses sont discutées sur les mécanismes qui pourraient présider à ces

Mots clés : Pin maritime, Pinus pinaster, Homoptera, Matsucoceus feytaudi, résistance naturelle.
\end{abstract}

\section{Introduction}

Une précédente note (Schvester, 1982) a fait part de premiers résultats d'observations sur les différences de sensibilité de diverses provenances de pin maritime (Pinus pinaster AIT.) à la Cochenille Matsucoccus feytaudi DUC. (Homoptera : Margarodidae).

Nous avions aussi, à cette époque, tenté de rechercher des explications aux différences observées :

- par une estimation des niveaux de population de l'insecte sur les diverses provenances,

- par un examen des caractères des tissus corticaux de certains arbres. 
Ceci n'avait pas été publié dans la note de 1982, assez succincte. De plus, les observations sur le comportement des diverses provenances ont été, depuis, poursuivies et nous proposons ici un examen d'ensemble des résultats.

Rappelons d'abord les grandes lignes du cycle de l'insecte: Matsucoccus feytaudi ne présente qu'une génération par an; les jeunes larves, mobiles, éclosent de fin mars à mi-mai, selon les caractères climatiques du lieu, se fixent une fois pour toutes, en implantant leurs stylets dans le liber, opèrent une première mue, en larves de deuxième stade, apodes, en septembre. La lignée mâle fournit, en décembre, des pronymphes, mobiles, puis des nymphes, enfin des adultes ailés dont la sortie, à partir de fin janvier - début février, coïncide avec celle des femelles, issues elles, directement sans autre stade intermédiaire, des larves de deuxième stade.

Matsucoccus feytaudi se comporte fondamentalement comme un insecte des troncs : les larves se fixent au fond des anfractuosités de ceux-ci, là où les "écailles" du rhytidome laissent entre elles des fissures où le liber se trouve à portée d'implantation des tylets. Les dégâts sur les extrémités des branches ( flagging ») sont liés à un niveau déjà élevé de pullulations sur tronc ou fortes branches.

\section{Matériel et méthodes}

Les observations ont pour cadre une plantation comparative mise en place en novembre 1965 avec plants 1.0. en godets dans le massifs de Lambert, en Forêt Domaniale des Maures, au lieu dit "Les Pounches» (coordonnées géographiques - en grades — 04.45 - 48.02) à altitude moyenne d'environ $520 \mathrm{~m}$, sous crête, en exposition Sud, avec pente moyenne d'environ 5 p. 100. L'implantation fut effectuée sur coupe rase, pratiquée l'été précédent, d'un peuplement âgé d'environ 30 ans, et en voie de destruction, précisément du fait de l'infestation par Matsucoccus feytaudi.

\subsection{Dispositif}

\subsection{Observations symptomatologiques}

Les provenances utilisées ont été au nombre de six (sans compter la provenance Maures) choisies a priori, parmi la gamme possible, pour les grandes différences dans leurs origines, à savoir : une provenance italienne (Gênes), une espagnole (Cuenca), une portugaise (Leiria), une corse (Marghese), une marocaine (Tamjoute), une landaise (Mimizan). Les graines étaient toutes issues de peuplements porte-graines répertoriés. Assez paradoxalement, il n'avait pas été possible de se procurer en temps utile de graines venant des Maures en quantité suffisante pour intégrer cette provenance dans le dispositif. Nous dûmes nous contenter d'installer au voisinage immédiat, une parcelle unique, de 75 arbres, de provenance Les Mayons (Var), à titre de référence. Nous avons utilisé comme référence supplémentaire des obscrvations effectuées d'autre part sur 50 arbres dominants d'un placeau immédiatement voisin, de même âge que la plantation, issu de régénération naturelle après la coupe rase.

Les 6 provenances non varoises ont été implantées selon un dispositif en blocs complets à 5 répétitions, chaque parcelle étant de $5 \times 5$ arbres plantés à $3 \times 3 \mathrm{~m}$. Un dispositif statistique n'étail pas en fait indispensable pour ce qui concerne l'éva- 
luation du comportement des arbres à Matsucoccus : celui-ci étant essentiellement propagé par les vents, à l'état de jeune larve, la probabilité d'être infesté est la même pour chaque arbre d'un peuplement homogène. Mais il avait été convenu, à l'occasion de cette plantation, de tenter d'acquérir aussi des données comparatives sur les caractères de la croissance et du développement des provenances.

L'ensemble du dispositif statistique était cerné de bordures sur une profondeur de 6 à 12 mètres selon le terrain, plantées à même distance $(3 \times 3 \mathrm{~m})$ d'arbres des différentes provenances. Les prélèvements d'arbres pour les divers examens ainsi que les expériences d'infestation de Carle relatées plus loin ont eu lieu sur ces bordures.

La plantation a beaucoup souffert dans ses deux premières années, nolamment par destruction de plants par Hylobius abietis ou par déterrage par des sangliers. Lés effectifs restants de chaque provenance sont néanmoins suffisants pour que les observations relatives au comportement à l'infestation par Matsucoccus puissent être considérées comme significatives.

\subsection{Méthodes de notation}

Chaque arbre, suivi individuellement, reçoit chaque année une note reflétant son degré de morbidité, et fondée sur une appréciation de l'intensité des symptômes d'infestation par Matsucocetus.

L'échelle de notation, un peu modifiée par rapport à celle publiée par CARus (1973) est la suivante :

Aucun symptôme

Symptcomes peu nets ef douteux sur un ou deux rameaux terminaux, atu plus

Quelques exsudations de résine sur le tronc et (ou) quelques rameaux terminaux atteints $\ldots \ldots \ldots \ldots \ldots \ldots \ldots$

Quelques écoulements de résine et moins du ticrs des ramcaux terminaux atteints ou détruits

Ecoulements de résine ef d'un tiers à la moitié des rameaux atteints ou détruits

Ecoulements de résine importants et plus de la moitié des rameatux atteints ou détruits

La presque totalité de la frondaison détruite

Arbre mort

(\%) Par rapport à l'échelle indiquée par CARLE nous avons rajouté cette note intermédiaire, pour tenir compte précisément du caractère douteux de certaines petites anomalies qu'il était cependant nécessaire de noter. 
Nous utilisons sur le terrain, l’échelle (1), l'échelle "transformée " (2) n'étant utilisée que pour l'interprétation des résultats.

De tout premiers symptômes étaient apparus sur quelques arbres à partir de 1965 , la plantation ayant alors dix ans. (Ce délai peut paraître long, mais nous savons, par de nombreuses observations sur des peuplements jeunes de régénération naturelle, qu'il est normal : les arbres jeunes n'ont pas l'écorce suffisamment craquelée ou fissurée pour permettre la multiplication de Matsucoccus à un niveau élevé, capable de provoquer des symptômes foliaires). Mais, pour éviter la formation d'idées préconçues, les notations détaillées n'ont été faites qu'à partir de 1979. Elles ont lieu au mois de mars, car c'est en fin d'hiver, et avant un nouveau départ de la végétation que les arbres extériorisent au mieux toutes les conséquences de l'évolution du " dépérissement " au cours de la saison précédente.

Elles ont toutes été effectuées par le même observateur (D. ScHvESTER) el chaque année sans référence à celles des années précédentes, ceci en vue, encore, d'éviter la formation d'idées préconçues.

\subsection{Expression des résultats}

Lcs résultats des relevés sont exprimés en pourcentage, dans chacun des degrés de l'échelle de notation, de l'effectif pris en compte pour chaque provenance (tabl. 1).

L'évolution d'ensemble sur la totalité de la période de notation, matéricllement difficile à traduire en tableaux est traduite graphiquement (fig. 2), pour chaque année et chaque provenance en termes de niveau global de morbidité atteint, selon la formule :

dans laquelle :

$$
(M)=\frac{T}{E \times 7} \times 100
$$

T. représente le total des notes (selon notre échelle "transformée »).

E. l'effectif pris en compte dans chacune des provenances,

7. la note maximale possible (arbre mort) dans l'échelle "transformée ".

S'agissant, en effet, de notations non paramétriques, il n'est pas possible d'exprimer les résultats par un total brut des notes. Nous les exprimons dans le graphique, en termes de pourcentages du total des notes par rapport à la note maximale possible $(E \times 7)$ pour chacune des provenances.

\subsection{Méthodes d'évaluation des niveaux de population de Matsucoccus}

\subsection{Piégeage de femelles}

La méthode est décrite par Carle: (1968), puis par Riom (1980) lequel fait en outre une analyse précise des conditions à réunir pour en assurer la fidélité. Rappelons seulement qu'elle met à profit le comportement des femelles fécondées : géotropisme positif et thygmotactisme. Des bandes de carton ondulé à cannelures de petites dimensions, écrasées à la presse et qui encerclent le fût de l'arbre, constituent le piège. Les bandes retirées, le dénombrement des insectes capturés a lieu aisément, par transparence, sur une surface vitrée éclairée par dessous. 
Cette méthode est non destructive, mais n'assure la capture que d'une partie de la population, dans une proportion inconnue par rapport à son niveau réel. Elle permet cependant des estimations relatives et des comparaisons entre arbres ou groupes d'arbres.

Pour assurer, autant que possible une homogénéité des conditions de capture, nous avons pris les précautions suivantes, recommandées par Rıom (1980) :

- choix d'arbres de dimensions comparables (ce qui. dans notre plantation, ne présentait aucune difficulté) ;

- pose des bandes non à hauteur uniforme, mais à la limite de fissuration des écorces, donc à hauteur légèrement variable selon les arbres;

- utilisation de bandes d'un même lot pour tous les arbres;

- piège constitué non d'une seule bande, mais de deux l'une au-dessus de l'autre, distantes d'environ $15 \mathrm{~cm}$.

Le piégeage a été effectué sur 30 arbres de chaque provenance, autant que possible à divers états de manifestation des symptômes et bien répartis dans l'ensemble de la plantation (pour la provenance Maures, sur la parcelle de "référence » pour la moitié, sur la placette de régénération voisine pour l'autre moitié).

Les pièges ont été mis en place le 4 février 1981 et maintenus jusqu'au 8 avril, période largement suffisante pour couvrir la totalité de celle d'activité des femelles adultes.

\subsection{Comptages}

Le comptage direct des populations sur l'arbre, largement utilisé par Rrom, permet une analyse précise des niveaux de population. Cependant la méthode est extrêmement contraignante et de plus, destructive. Pour des raisons matérielles nous n'avons effectué de comptages que sur trois arbres, tous sans symptômes, de chacune des provenances Cuenca et Tamjoute, arbres prélevés hors dispositif, dans les bordures. Nous pensons potivoir considérer que les résultats antérieurs acquis par Riom sur les arbres des Maures, constituent une référence suffisante, à défaut d'un témoin au sens strict du terme.

Ces comptages ont été effectués en novembre 1982, époque où l'insecte se trouve au deuxième stade larvaire. Ils n'ont intéressé que la partie fissurée ou écailleuse des troncs soit environ $2 \mathrm{~m}$ par individu.

Ces troncs, ramenés au laboratoire, sont coupés en tronçons de $25 \mathrm{~cm}$ chacun. Un tronçon sur deux, en alternance sur toute la hauteur, est l'objet du dénombrement, moyennant décortication soigneuse sous loupe binoculaire.

\subsection{Etude de la structure des tissus corticaux}

Les observations sur les populations suggéraient que le tronc des provenances Tamjoute et Cuenca paraît impropre à la fixation des cochenilles en nombre important. 
Nous avons tenté de rechercher des explications à ces faits dans d'éventuelles différences dans la structure et dans l'agencement des tissus végétaux au niveau des points d'implantation des insectes, ceci à l'échelle microscopique d'une part, macroscopique de l'autre.

\subsection{Etude microscopique}

Nous avons examiné :

- d'une part, sur les rameaux terminaux, la région située à la jonction de la pousse de l'année précédente. Les prélèvements ont été effectués en novembre 1980 , hors de la période de végétation à raison de 6 rameaux par arbre à différents niveaux, à raison de 5 arbres sur chacune des provenances Maures, Cuenca et Tamjoute. Puis un nouvel examen fut effectué en avril, à la reprise de la végétation à raison de 6 rameaux par arbre sur 3 arbres de chacune des mêmes provenances;

- d'autre part des prélèvement à l'emporte-pièce de fragments de rhytidome dans la zone de contact entre liber et périderme, au niveau des fissures au fond desquelles s’implante la cochenille (fig. 1). Les échantillons ont été prélevés à trois niveaux du fût, en décembre sur deux arbres de chacune des provenances Maures, Cuenca et Tamjoute, puis en avril, mais à un seul niveau sur trois autres arbres de chacune des mêmes provenances.

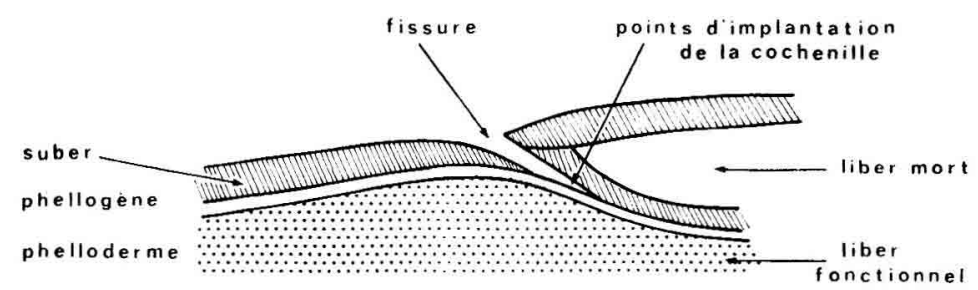

Fig. 1

Représentation schématique en coupe transversale du décollement du rhytidome de pin maritime au niveau d'une écaille.

Schematic representation of the detachment of cluster pine rhytidome transversal section trough a bark-flake.

Les coupes sont faites au microtome à congélation puis, après passage clans une solution d'hypochlorite de sodium, colorées soit at carmin-vert d'iode, soil au Soudan III.

\subsection{Etude macroscopique}

Par suite de la croissance en épaisseur de la tige le rhytidome se fissure et se craquelle. D’où sa structure "écailleuse".

Sur le tronc , les larves de Matsucoccus se fixent et sont concentrées uniquement le long des «lignes» de décollement de ces écailles du rytidome, aux endroiis où le clivage entre liber et écorce se produit de façon telle que, l'écaille ayant entrainé 
toutes les couches de suber lors du décollement, une étroite frange de liber se trouve directement à portée des stylets.

La coupe (fig. 1) schématise ce décollement au niveau du phellogène et du phelloderme. A la lisière de la zone de décollement, la région libérienne fonctionnelle n'est plus protégée que par une portion de phelloderme peu épais et plus ou moins déchiré. Le suber est également interrompu au niveau de la fissure laissant ce que nous appelons plus loin, une mince "frange colonisable»: la cochenille peut ici atteindre facilement le liber en se glissant au fond de la fissure, sous l'écaille.

Il est aisé d'observer de tels décollements en faisant sauter les écailles du rhytidome, ceci du moins lorsque la colonisation par Matsucoccus n'a pas encore abouti à un colmatage des fissures, et nous avons tenté de quantifier par ce moyen les possibilités ainsi offertes pour la fixation et la survie des cochenilles. A cet effet nous soulevons soigneusement les écailles à l'aide d'un fort couteau en évitant de déchirer les tissus. Cet écorçage ne peut concerner que les zones fissurées ou squameuses. Mais cette limitation est de peu d'importance puisque les zones non fissurées ne peuvent héberger que de faibles populations de cochenilles;

Les plages de liber ainsi mises à nu sont mesurées dans leur plus grande longueur et nous considérons que cette longueur - multipliée par deux puisque des larves peuvent se fixer des deux côtés de l'écaille - correspond à la longueur de frange colonisable.

La longueur totale pour chaque arbre est rapportée à la surface sous écorce.

Ont été traités, 5 arbres de provenance Tamjoute, et 5 de provenance Cuenca indemnes de symptômes, 3 arbres de provenance Maures issus de la plantation et n'exprimant que des symptômes légers, auxquels nous avons ajouté 2 arbres de même àge sans symptômes, prélevés en un autre site.

\section{Résultats}

\subsection{Les symptômes selon les provenances}

Le tableau 1 donne la répartition des individus des diverses provenances, dans les divers degrés de notre échelle de notation. D'une part, d'après les notations de mars 1985 (reflétant par conséquent l'état des arbres après la saison 1984) d'autre part. à titre de comparaison d'après les notations de mars 1982 (reflétant l'état des arbres après la saison 1981) antérieurement publiées.

Il ressort clairement, que se distinguent des autres, les provenances Tamjoute (Maroc) et Cuenca (Espagne-centre). Même si, certaines années, quelques symptómes ont été notés, sur quelques rares arbres de ces provenances il s'agit dans la plupart des cas de symptômes douteux, relevés par scrupule, ou de symptômes très légers, réversibles, et dont nous avons observé qu'ils n'affectaient que rarement les mêmes sujets deux années consécutives.

La provenance Gênes est apparue au moins aussi sensible que les pins des Maures, ce qui ne paraît pas étonnant. Divers auteurs (Sweet \& Thulın, 1963, Destremau 


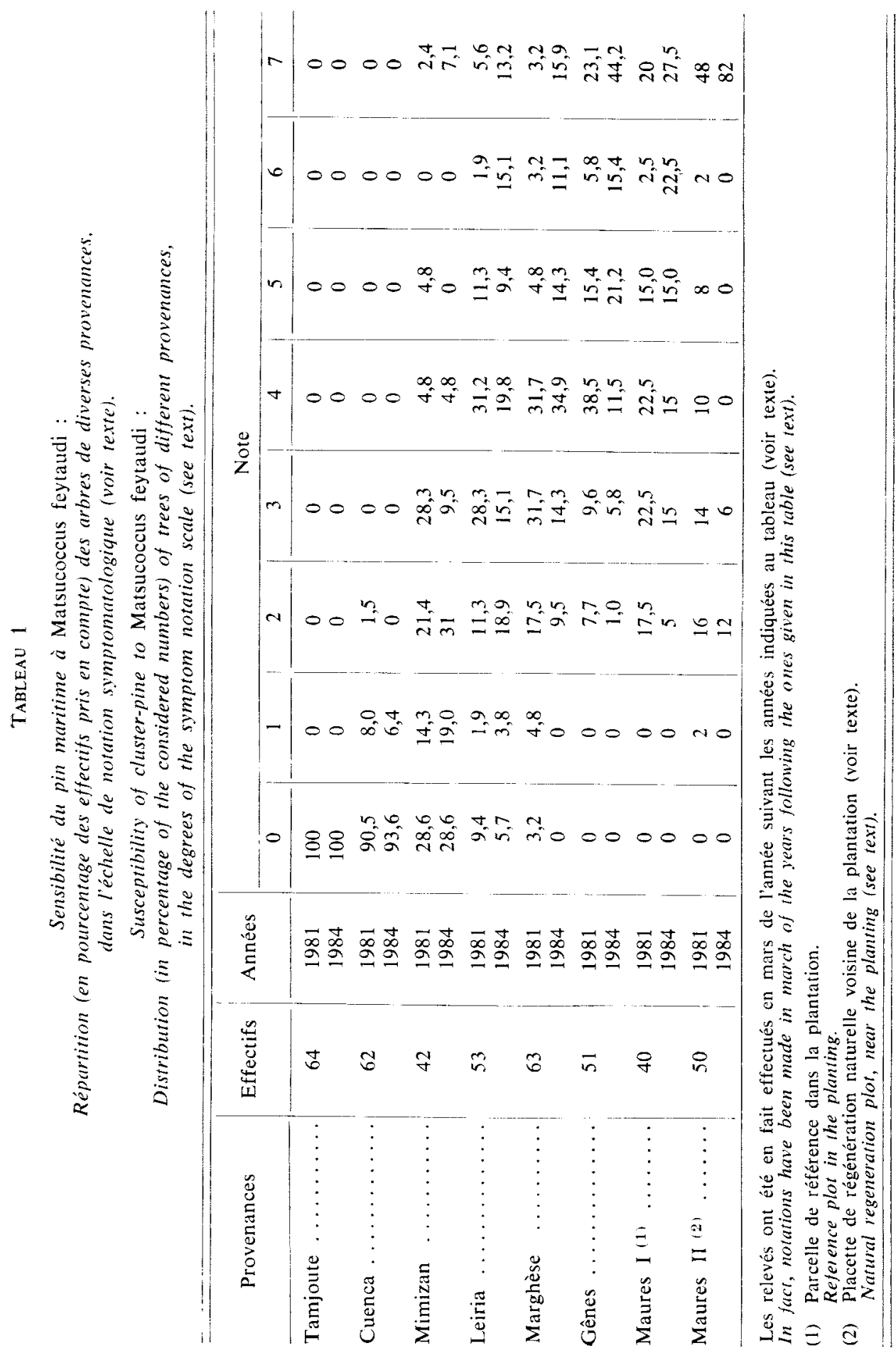


et al., 1976, entre autres) considèrent les provenances ligures comme extrêmement voisines des provenances méditerranéennes françaises. Les analyses terpéniques montrent qu'il s'agit effectivement de la même population ou de populations très voisines (BARADAT, communication personnelle). On sait, au reste, que l'infestation par $M$. feytaudi se propage actuellement en ltalie, avec des conséquences analogues à ce qui s'est passé en France (FABre ,1980).

La provenance Marghese apparaît, dans les conditions de l'expérience, un peu moins sensible, actuellement, que les provenances Maures ou Gênes mais suffisamment cependant pour laisser craindre de graves conséquences si $M$. feytaudi venait à être introduit en Corse.

Les provenances atlantiques Mimizan (Landes) et Leiria (Portugal) apparaitraient relativement moins sensibles, la première nommée surtout, avec cependant une pro-

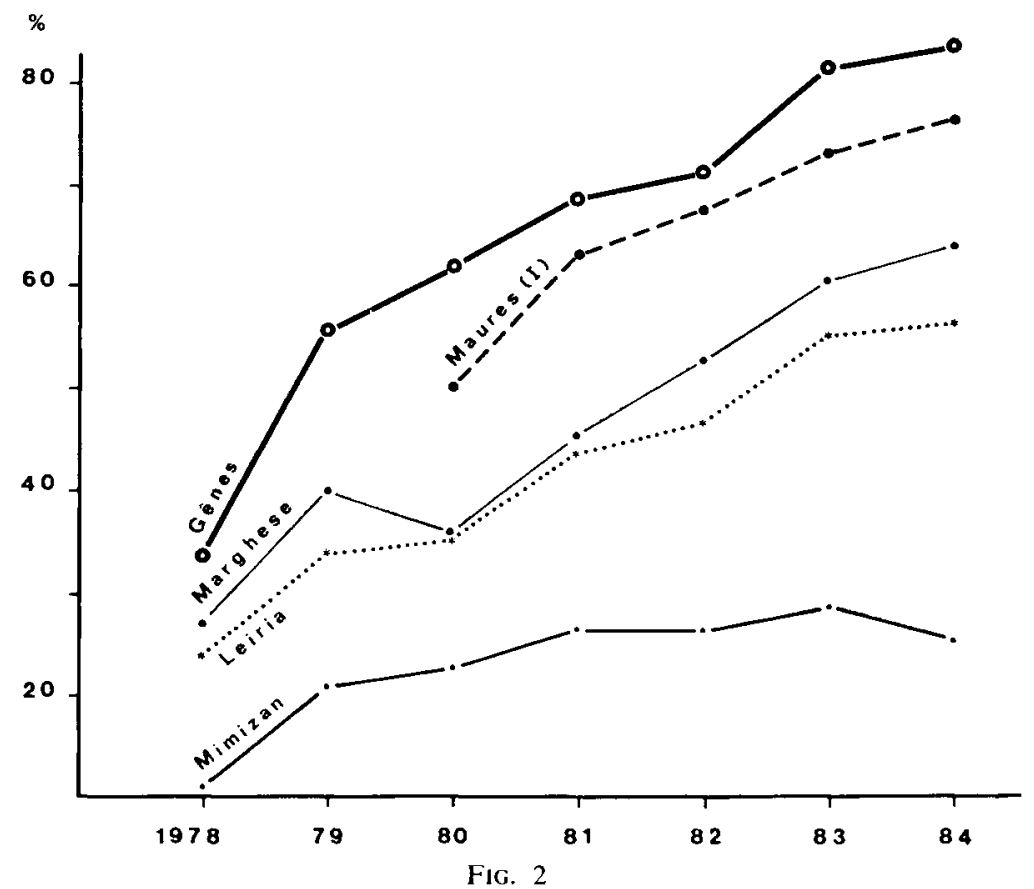

Progression des symptômes de dépérissement dus à Matsucoccus feytaudi sur diverses provenances du pin maritime.

Progression of symptoms of die-back due to Matsucoccus feytaudi on diverse provenances of cluster-pine.

En abscisse : Les années indiquées sont celles auxquelles se rapportent les observations, et non celles des observations elles-mêmes qui ont eu lieu en mars de l'année suivante (voir texte).

En ordonnée : Pourcentages du total des notes de symptômes observées par rapport a la note maximale possible pour chaque provenance (effectif d'arbres pris en compte $\times 7$ ).

Abscissa : The given years are those which the observations are related to, not those of the observations themselves, occurring in March of the following years (see text).

Ordinate: Percentage of the observed symptom notes, related to the maximal possible note for each provenance (number of considered trees $\times 7$ ). 
portion déjà élevée d’arbres pouvant présenter des symptômes assez avancés. On observe cependant un «tassement» en 1984 par rapport aux années antérieures.

Le classement par ordre de sensibilité tel qu'il découle de ces observations, est resté le méme chaque année. En outre, pour les provenances autres que Tamjoute et Cuenca, on observe globalement, une aggravation annuelle presque constante des symtômes même si, dans quelques cas, on a pu, sur certains individus observer une régression certaines années par rapport à la précédente. Ceci est pour partie lié au caractère subjectif des notations et n'a concerné qu'un nombre réduit d'arbres, affectés le plus souvent de notes faibles, la régression observée n'étant que d'un point, et beaucoup plus rarement de deux.

Le taux d'aggravation selon les provenances respecte également, à très peu de chose près, le même classement et peut d'ailleurs varier : il est important en 1980 par rapport à 1979, nettement moins important en 1981 par rapport à 1980 où l'on observe même une certaine stabilisation. Laggravation reprend en 1982, et surtout en 1983. Nous pensons qu'il faut voir là, l'effet des conditions climatiques et surtout :

- celles du printemps précédent l'observation, la pluviosité de cette période conditionne en partie la survie des larves de la cochenille, de même que les possibilités de fécondation des femelles (RıOM \& Fabre, 1970 ; Riom, 1980);

- celles aussi de l'été el de l'automne précédant l'observation, dans la mesure où les élé chauds et secs favorisent la multiplication de $P$. notatus (aggravant ainsi la mortalité) et où conjointement, elles amoindrissent les capacités de réaction des arbres.

\subsection{Niveaux de population de Matsucoccus selon les provenances}

\subsection{Résultals des piégeages de femelles}

Ces résultats sont fournis au tableau 2.

En dépit des imperfections de la méthode, il est très net que les captures sur les provenances Tamjoute et Cuenca sont très significativement moindres que sur les autres provenances.

A l'intérieur de chaque provenance (sauf Tamjoute et Cuenca), la variation du nombre de femelles capturées est importante. Mais ceci correspond à toutes les observations faites en d'autres station (CARle, 1973 ; RaOul DE Pontivy, 1978).

On n'a pas observé de rapport net, arbre par arbre, entre le nombre d'insectes capturés et la note de symptômes, et le classement des provenances selon les quantités d'insectes capturées ne reflète pas exactement celui qui découle des notations de symptômes.

En outre, les effectifs capturés peuvent paraître dans l'ensemble assez faibles si on les compare aux chiffres fournis par CARLE (1968) ou par RIOM (1980). Ceci peut être attribué au fait que probablement certains arbres se trouvaient déjà en cours d" "autorégulation " au sens de Riom. Rappelons que ce phénomène découle de ce que la survie et la multiplication de l'insecte sont subordonnées à l'existence de tissus de liber frais dans lequel il peut implanter ses stylets pour se nourrir; 
les tissus lésés par des implantations antérieures ne sont plus «colonisables » et if en résulte, après quelques générations, un abaissement assez net des niveaux de population.

\section{TABleav 2}

Nombre de femelles de Matsucoccus feytaudi capturées par bandes-pièges sur les diverses provenances (du 4 jévrier alu 8 avril 1981 sur 30 arbres par provenance).

Numbers of Matsucoccus feytaudi female trapped by «strip traps on the different provenances (from 4th Febrtary to 8th April 1981, on 30 trees per provenance).

\begin{tabular}{|c|c|c|c|c|c|}
\hline Provenance & $\begin{array}{c}\text { Total } \\
\text { des femelles } \\
\text { capturées }\end{array}$ & Minimum & Maximum & $\begin{array}{l}\text { Moyenne } \\
\text { par arbre }\end{array}$ & Ecart type \\
\hline Tamjoute & 116 & 0 & 9 & 3,86 & 3,03 \\
\hline Cuenca. & 171 & 0 & 16 & 5,7 & 4,48 \\
\hline Mimizan & 1379 & 11 & 230 & 45,96 & 42,38 \\
\hline Leiria & 1034 & 6 & 121 & 34,46 & 24,46 \\
\hline Marghèse & 1134 & 6 & 75 & 37,8 & 19,18 \\
\hline Gênes ... & 1265 & 6 & 192 & 42,16 & 47,12 \\
\hline Maures & 976 & 4 & 93 & 32,53 & 23,21 \\
\hline
\end{tabular}

Mais quoi qu'il en soit, les différences à cet égard entre les provenances espagnole et marocaine d'une part, et les autres provenances d'autre part, restent considérables.

\subsection{Comptages}

Les résultats sur les six arbres examinés sont donnés ci-après :

\begin{tabular}{|c|c|c|c|c|}
\hline \multicolumn{2}{|c|}{ Arbre } & $\begin{array}{l}\text { Surface }\left(\mathrm{m}^{\prime \prime}\right) \text { examinée } \\
\text { (sous écorce) }\end{array}$ & $\begin{array}{c}\text { Nombre de larves (L II) } \\
\text { (vivantes) }\end{array}$ & Densité par $\mathrm{cm}^{2}$ \\
\hline Cuenca & 1 & 0,72 & 11 & 0,0015 \\
\hline Cuenca & 2 & 0,58 & 7 & 0,0012 \\
\hline Cuenca & 3 & 0,77 & 0 & 0 \\
\hline Tamjoute & 1 & 0,65 & 2 & 0,0003 \\
\hline Tamjoute & 2 & 0,80 & 6 & 0.00007 \\
\hline Tamjoute & 3 & 0,67 & 4 & 0,0006 \\
\hline
\end{tabular}

Ces chiffres sont à confronter aux données de Rıom et al. (1971) selon lesquelles, sur les arbres des Maures, les densités de larves de deuxième stade (d'ailleurs assez variables) atteignent jusqu'à 10 par $\mathrm{cm}^{2}$ de tronc fissuré. 


\subsection{Observations sur les tissus corticaux}

\subsection{Etude microscopique}

Les coupes de rameaux n'ont laissé apparaître aucune particularité anatomique chez aucune des provenances, ni, par exemple, une épaisseur plus importante de suber, ni sclérenchyme entre suber et liber.

De même, aucune particularité n'a été décelée sur les coupes effectuées au niveau du rhytidome.

Il ne semble pas exister de différences anatomiques notables susceptibles d'induire chez l'une ou l'autre des provenances, une " résistance mécanique " à l'implantation de la cochenille.

\subsection{Etude macroscopique}

Les résultats en sont résumés au tableau 3. Malgré la grossièreté et l'imprécision du procédé, de nettes différences apparaissent entre les provenances. Les pins de provenance Maures, aussi bien ceux de la plantation que ceux de l'autre site présentent une longueur colonisable plus importante en moyenne que celle offerte par les provenances Tamjoute et Cuenca. La variabilité semble assez importante à l'intéricur de cette dernière mais, étant donné les imperfections de la méthode, aucune conclusion ne peut être tirée. Il semble toutefois assez net que les provenances Tamjoute et Cuenca offrent aux cochenilles beaucoup moins de sites propices à leur fixation et leur développement que la provenance Maures.

\section{TABLEAU 3}

\section{Possibilités d'établissement de Matsucoccus feytaudi} sur certaines provenances de pin maritime.

Mesures de la longueur de «franges colonisables» (5 arbres par provenance).

Possibilities of settlement of Matsucoccus feytaudi on certain provenances of cluster-pine. Mesures of lenght of "colonizable bands» $(5$ trees per provenance).

\begin{tabular}{c|c|c|c}
\hline Provenance & $\begin{array}{c}\text { Longueur colonisable } \\
\text { total/arbre }(\mathrm{cm})\end{array}$ & $\begin{array}{c}\text { Surface étudiée } \\
\left(\mathrm{m}^{2}\right)\end{array}$ & $\begin{array}{c}\text { Longueur colonisable } \\
(\mathrm{cm}) / \mathrm{m}^{2} \text { de surface }\end{array}$ \\
\hline MAROC & 68 & 0,8011 & 84,88 \\
(Tamjoute) & 86 & 0,8011 & 107,35 \\
& 42 & 1,0600 & 39,62 \\
& 65 & 0,6440 & 100,93 \\
73 & 0,6440 & 113,35 \\
ESPAGNE & 171 & 0,5733 & 298,62 \\
(Cuenca) & 332 & 1,0210 & 325,66 \\
& 38 & 0,5340 & 71,16 \\
& 28 & 0,6048 & 46,29 \\
& 22 & 0,7697 & 28,58 \\
MAURES & 622 & 0,9346 & 665,52 \\
& 325 & 0,8875 & 366,19 \\
& 639 & 0,7932 & 427,38 \\
& 526 & 0,9346 & 568,23 \\
& & 0,7618 & 400,71 \\
\hline
\end{tabular}




\section{Discussion et conclusion}

En l'état actuel des observations, alors que les arbres atteignent leur vingtième année, se confirment effectivement d'importantes différences entre les provenances de pin maritime mises en expérience, dans la sensibilité à Matsucoccus feylaudi. MENDEL (1984) constate également des différences selon les provenances dans la sensibilité de Pinus halepensis à Matsucoccus josephi mais semble-t-il, moins tranchées.

Si l'on se rapporte aux résultats des piégeages de femelles et des comptages de larves, il semble ressortir une relation assez nette entre l' "immunité » des provenances Tamjoute et Cuenca, et leur apparente incapacité à permettre à Matsucoccus de s'y multiplier jusqu'à des niveaux générateurs de dommages (dans les conditions de l'expérience du moins).

Or, Riom (1980) conclut au contraire « qu'en aucun cas, la provenance des pins n'est l'élément déterminant de la pullulation de $M$. feytaudi". Cette conclusion est fondée, essentiellement sur des observations et des expériences effectuées dans les Landes, sur une plantation comparative (forêt des Malgaches). Il fait état, en outre, d'observations sur une plantation comparative à Cuntis (Espagne, Province de Pontevedra en Galice) et sur la forêt de Lambert sur cette même plantation ici en cause. Concernant Cuntis, il indique, sans autres détails que les «provenances Landes, Maures et Estérel hébergent des populations naturelles de M. Feytaudi de niveaux comparables ». Concernant Lambert, il s'agit de constatations d'ordre général sur le comportement des arbres, antérieures à 1975, donc avant que ces arbres encore jeunes aient exprimé de quelconques symptômes.

Concernant donc la plantation des Malgaches, observons que :

- Rıom ne fait état que des provenances Maures, Estérel, Landes et Portugal. Et il observe par exemple que par piégeage les effectifs de femelles capturées sur les provenances Maures et Estérel sont plus importants que sur les provenances landaise et portugaise (par ex. moyenne sur 20 arbres 4,7 pour la provenance landaise contre 26 pour la provenance Maures). Il n'a cependant, et avec raison, voulu interpréter ces résultats qu'avec une grande prudence, compte tenu des conditions de l'expérience d'une part, compte tenu d'autre part des résultats d'autres observations;

- les résultats sur la comparaison de la multiplication de l'insecte selon les provenances, ont pu être masqués par l'influence des conditions climatiques landaises sur les possibilités de cette multiplication. Rıom démontre en effet, expérimentalement un rôle important de l'humidité atmosphérique dans la limitation des populations dans les Landes. Mais dans les conditions naturelles, même à la suite d'infestations forcées massives, par apport de pontes (jusqu'à 1000 pontes par arbre) la densité de larves dénombrées l'automne suivant au deuxième stade demeure très faible : $1 \mathrm{~L} 2$ au $\mathrm{cm}^{2}$ d'écorce au plus, contre jusqu'à 10 dans les Maures, des dommages importants pouvant survenir à partir de $5 \mathrm{~L} 2$ au cm".

Si l'on admet, au moins par hypothèse, une relation effective entre l' "ummunité " de certaines provenances et l'impossibilité pour la cochenille de s'y multiplier jusqu'à des niveaux générateurs de dommages c'est qu'il existe de la part de ces provenances, une "antibiose» (antibiosis) au sens de Painter (1958) à l'égard de la cochenille. La question reste posée des mécanismes qui président à cette antibiose, la notion, assez 
vague de «bonne adaptation au milieu " avancée par CHARARAS (1977) à propos du bon comportement à Matsucoccus des pins marocains au Maroc n'étant guère explicative.

Les causes premières de cette "antibiose " peuvent être diverses. Nous pensons pouvoir admettre l'existence d'un facteur "mécanique". D'ailleurs, selon une hypothèse précédemment émise par Riom (in Schvester et al., 1970) «pourrait intervenir la constitution morphologie des arbres, selon que. par le caractère et l'intensité de la fissuration de leurs écorces, ils offrent des possibilités d'implantation à un nombre plus ou moins important de larves" '11. Or, dans notre plantation, l'agencement des tissus corticaux du tronc et les caractères de leur exfoliation chez les provenances "Tamjoute » et "Cuenca » ne laissent effectivement que peu de «franges colonisables » de liber, ce qui doit bien constituer un frein à la multiplication de l'insecte.

Cependant, sur ces provenances il s'en faut, et de beaucoup, que les « franges colonisables » même moins nombreuses soient totalement colonisées. Ceci peut suggérer l'intervention d'autres facteurs, d'origine physiologique ou biochimique, auxquels on peut supposer une origine génétique.

A l'appui de cette hypothèse, rappelons le résultat des obscrvations de Carle (1973) sur les réactions des tissus d'extrémités de rameaux à des expériences d'infestation forcée sur quelques arbres de chaque provenance de notre plantation, les arbres étant encore jeunes (six ans) : CARLE observe sur «Tamjoute», une rapide différenciation d'assise subéro-phellodermique qui permet souvent le rejet des tissus lésés par les piqûres; sur "Cuenca », de très fortes réactions sous-épidermiques qualifiées de cécidogènes; des réactions cecidogènes moins vives sur "Mimizan " et «Lciria»; des réactions très peu marquées autour des lésions primaires dues aux piqûres sur les provenances "Maures », "Gênes" et "Marghese ». S'agissant ici d'infestations à densité élevée de larves, des lésions sont observées dans tous les cas, mais les observations témoigneraient bien de potentialités de réactions d'origine physiologique différentes selon les provenances alors même que les rameaux intacts ne présentent pas de différences anatomiques décelables.

Le facteur "mécanique " (structure et agencement des tissus corticaux) pourrait d'ailleurs, lui aussi, être d'origine génétique, tout en étant peut-être régi aussi par certains facteurs du milieu (édaphiques, climatiques). Rıom (communication personnelle) a d'ailleurs bien constaté que la structure des écorces de la provenance "Maures" dans la plantation des Malgaches était différente de ce qu'elle est dans les Maures. Cadahia \& Montoya (1968) font la même constatation dans la plantation de Cuntis : «Les blocs de provenance « Maures " n'y subissent qu'une faible attaque de Matsucoccus... et les caractères de leurs écorces sont très différents à Cuntis... l'écorce est plus épaisse et offre moins de possibilités d'installation que dans les Maures».

En résumé, les facteurs qui peuvent régir la sensibilité des pins maritimes à Matsucoccus apparaîtraient complexes :

- facteurs génétiques gouvernant, indépendamment ou simultanément les caractères physiologiques et/ou biochimiques du végétal, et les caractères anatomiques (structure des écorces);

- facteurs phénotypiques gouvernés par les conditions (édaphiques, clima-

(1) Voir aussi dans le même numéro de la Revue Forestière Française, au chıpitre « Débats », p. 293. 
tiques...) du milieu, dans la mesure où celles-ci peuvent influer sur certains caractères (physiologiques, anatomiques...) du génotype.

Ceci doit inciter à une certaine prudence dans un éventuel développement. Jusqu'à présent, il ne s'agit que d'une seule et unique expérience et il convient de chercher à en vérifier les résultats dans des conditions différentes, par exemple aux basses altitudes. Il ne peut être a priori exclu, par exemple sur "Cuenca ", que les symptômes extrềmement bénins à $500 \mathrm{~m}$ d'altitude, soit aggravés en plaine. Nous connaissons ainsi à la Môle (alt. $50 \mathrm{~m}$ environ) une plantation d'environ 30 ans, d'arbres d'origine landaise (en fait : Villandraut, Gironde), dont beaucoup d'arbres ont survécu, mais la plupart de ces survivants sont plus ou moins tarés en particulier par des attaques de Dioryctria, alors que les survivants landais de notre plantation paraissent pour la plupart en bien meilleure condition. Pour un avenir plus lointain, se posent également les problèmes découlant des possibilités d'hybridation entre sujets survivants des Maures et les individus d'autres provenances qui pourraient être introduits, donnant des descendances dont il n'est pas possible actuellement de prévoir le comportement à l'infestation par Matsucoccus.

Reçu le 8 novembre 1985. Accepté le 15 mai 1986.

\section{Remerciements}

Nous remercions pour son aide matérielle lors de la mise en place de la plantation, l'Office National des Forêts, en la personne surtout de M. Borssıs, alors Chef du Centre de l'O.N.F. de Toulon, décédé depuis. M. B. Gerbinot et M. Ferrandes qui ont largement contribué à la mise en place, puis à certaines observations.

\section{Summary \\ Differences in susceptibility to Matsucoccus feytaudi DUC. (Homoptera : Margarodidae) in cluster pine (Pinus pinaster AIT.) according to provenance.}

\footnotetext{
Observation were made on the susceptibility of some provenances of cluster pine (Pinus pinaster AIT.) to infestation by Matsucoccus feytaudi DUC., in a comparative plantation set up in 1965 in the Maures-Hills (provence Southern France).

Observations consisted of :

- symptom-notations tree by tree, carried out from 1979 to 1984 ;

- a search for an explanation of the observed differences, through, on the one hand, an evaluation of the insect population level (by trapping females and by counting second instar nymphae). On the other hand, in certain provenances only, through an examination of the character of the bark-tissues.

Symptom-notations (table 1 and figure 1), point to important differences between the tested provenances regarding susceptibility to $M$. feytaudi.

Under the conditions of the experiment, provenances from Cuenca (Spain) and from Tamjoute (Morocco) appeared symptom-free (or almost symptom-free, for the first one), other provenances : Mimizan (Landes, France), Leiria (Portugal), Marghese (Corsica, France), Genova (Italy) and Maures (France) were affected to different degrees, the two latter being very heavily affected.
} 
The results of trapping (table 2) and counting (see in text 3.22.) suggest that the immunity of the Cuenca and Tamjoute provenances seems to be related to the impossibility for Matsucoccus to multiply to important and injurious levels on these provenances.

The observations, on the bark, and other previous observations (CARLI:, 1973; RIOM, 1980) suggest different hypotheses for the mechanisms which might govern these differences.

- Mechanical factors, such as the structure of the bark which seems to constitue an obstacle for the settlement of the crawlers. They might be induced genetically and/or by environmental conditions (edaphic, climatic...). induced.

- Physiological and/or biochemical factors of the host plant, possibly genetically

Key words : Cluster pine. Pinus pinaster. Homoptera, Matsucoccus feytaudi, natural resistance.

\section{Références bibliographiques}

Cadahia D., Montoya R., 1968. Prospeccion de Matsucoccus feytaudi DUC. y Matsucoccus pini GREEN (Homoptera : Margarodidae) en la Mitad Norte de España. Bol. Serv. Plagas For., 22, 133-134.

Carle P., 1968. Méthode d'obtention massive des pontes de Matsucoccus feytaudi par piégeage des femelles. Ann. Sci. For., 25 (2), 57-68.

Carle P., 1973. Le dépérissement du pin mésogéen en Provence. Rôle des insectes dans les modifications d'équilibre biologique des forêts envahies par Matsucoccus feytaudi $D U C$. Thèse Doct. Sciences Nat., Bordeaux I, n" 377, 174 p. + Biblio.

Destremau D.X., Jolly H., Tahri T., 1976. Contribution à la connaissance des provenances de Pinus pinaster. Ann. Rech. For. Maroc, 1976, 16, 101-153.

FABRE J.P., 1980. Mortalité dans les peuplements de pin maritime à la suite de l'introduction de Matsucoccus feytaudi DUC. en Italie. L'Italia Forestale e Montana, 35 (1), 41-42.

Chararas C., 1977. Problèmes posés dans différents pays méditerranéens par les insectes parasites des Forêts. II. Aperçu général sur les insectes forestiers dı Maroc. C.R. Acad. Agr. Fr., 63 (10), 611-617.

Mendel Z., 1984. Provenance as a factor in susceptibility of Pinus halepensis to Matsucoccus josephi (Homoptera : Margarodidae). For. Ecol. Manage., 9, 259-266.

Painter H., 1958. Resistance of Plants to Insects. Ann. Rev. Entomol., 3, 267-290.

RaOul de POntivy G., 1978. Contribution à l'étude de l'évolution dans les peaplements méditerranéens de pin maritime du dépérissement dî à Matsucoccus feytaudi DUC. et des biocoenoses associées de ravageurs subcorticaux. Thèse doctorat d'Université Aix-Marseille III, $191+10$ p. + annexes.

Riom J., Gerbinot B., Boulbria A., Fabre J.P., 1971. Eléments de la bioécologie de Matsucoccus feytatudi DUC. et de ses prédateurs dans le Sud-Est et le Sud-Ouest de la France. Ann. Zool. Ecol. Anim. $N^{\prime \prime}$ spécial «La lutte biologique en forêt 》, 153-176.

RıM J., Fabre J.C., 1970. Décalage phénologique des éclosions des deux sexes chez Matsucoccus fevtaudi DUC. (Homoptera, Coccoidea, Margarodidae). C.R. Acatl. Sc. Paris, 288, ser. D. 89-92.

Rrom J., 1980. Biologie et écologie des populations de la cochenille du pin maritime Matsucoccus feytaudi DUC. 1942 (Coccoidca, Margarodidae). Thèse Doct. Sciences Nat. Univ. Bordeaux I, n" 660, 253 p. + Biblio.

Schvester D., Carle P., Riom J., 1970. Le dépérissement du pin maritime dans le Var. Etat actuel du problème "Matsucoccus fevtaudi». Rev. For. Fr. N" spécial "La lutte biologique en forêt », 240-246.

SCHVESTER D., 1982. Incidence de Matsucoceus feytaudi DUC, sur pins maritimes de diverses provenances (Pinus pinaster AIT.) en région méditerranéenne. C.R. Acad. Agr. Fr., 68 (9), 1324-1333.

Sweet G.B., Thulin I.J., 1963. Provenance of Pintis pinaster AIT. A five year progress report on a trial at Woodhill N. Zealand. New Zeal. J. For., VIII (4), 570-585. 